Pacific Journal of Mathematics

A NOTE ON LINEARLY ORDERED NET SPACES 


\title{
A NOTE ON LINEARLY ORDERED NET SPACES
}

\author{
JAMES R. BOONE
}

The linearly ordered net spaces are introduced in this paper. This concept is a generalization of both the sequential spaces and the linearly ordered base spaces. The fundamental properties of this class, including mapping properties, are presented. Various applications of a general nature are given as well as some applications to weak covering axioms. The class of lo-net spaces is characterized as the class of well-ordered net spaces.

I. Introduction. In this note the large class of linearly ordered net (lo-net) spaces is introduced, the fundamental properties of this class are presented and the study of applications is initiated. The lo-net spaces are very useful simultaneous generalizations of sequential spaces and linearly ordered base spaces. The applications of sequential spaces are numerous and well known. The linearly ordered base (lob) spaces were studied by Davis [6] and applications of a general nature were presented along with the beginnings of important applications to weak covering axioms. In [7] the applications of lob-spaces to various weak covering axioms were studied extensively.

Section 2 contains the definitions of the notions associated with the linearly ordered net spaces, as spaces with the weak topology generated by a class of subsets. The fundamental structural properties and some of the basic applications are presented in $\S 3$. In $\S 4$ some of the results of [6] and [7] are reexamined from the broadened view of lo-net spaces. The subtle differences between lo-net and very lo-net spaces are illustrated by examples. In particular, Lemma 2.3.1 of [7] is not true and a corrected version is presented along with its lo-net version. Finally the true nature of the lo-net spaces as a generalization of sequential spaces and lobspaces is revealed in $\S 5$, where the class of lo-net spaces is characterized as the class of well-ordered net spaces.

II. Preliminaries. A linearly ordered net (lo-net) is a net whose directed set is linearly ordered. The collection of linearly ordered nets $\left(D_{X}\right.$ in a space $X$ determines a natural cover [9] of $X$. Many of the notions in this study are specific applications of concepts and properties introduced and developed by Stan Franklin [9]. A topological space $X$ will be called a linearly ordered net (lo-net) space provided $H \subset X$ is closed if and only if for every convergent lo-net in $H$, say $x_{\lambda} \rightarrow x$, we have $x \in H$. That is $X$ is a lo-not space 
provided $X$ has the weak topology determined by the collection (D) . $^{2}$ The class of lo-net spaces is very large including all sequential spaces and lob-spaces. (A space is a lob-space provided every point has a nhood base which is linearly ordered by set inclusion [6].) The lob-spaces and results of [6] are the primary motivation for this work. The terminology used by Arhangelskii, very $k$-space, is quite indicative of the property described and I use it as follows: $X$ is a very lo-net space if for each $x \in \mathrm{cl}(H)$ there exists a convergent lo-net $\left(x_{\lambda}\right)$ in $H$ such that $x_{\lambda} \rightarrow x$. For each $A \subset X$, let lo-cl $(A)=\{p: p$ is the limit of a convergent lo-net in $A\}$. Recall the essential subtle point that lo-cl $(A)$ is not necessarily closed, even in a lo-net space. For each $A \subset X$, let $A^{0}=A, A^{1}=\operatorname{lo-cl}(A)$, for any fixed ordinal $\beta$ where $A^{\alpha}$ is defined for each $\alpha<\beta$, if $\beta=$ $\gamma+1$, define $A^{\beta}=\operatorname{lo}-\mathrm{cl}\left(A^{\gamma}\right)$ and if $\beta$ is a limit ordinal let $A^{\beta}=$ $\cup\left\{A^{\alpha}: \alpha<\beta\right\}$. The lo-net characteristic of a space $X, \lambda(X)$, is the least ordinal $\alpha$ such that $A^{\alpha}=\operatorname{cl}(A)$, for each $A \subset X . \quad X$ is a lo-net space if and only if $\lambda(X)$ exists, and in this case $\lambda(X) \leqq(t(X))^{+}$ where $t(X)$ is the tightness of $X . \quad X$ is a very lo-net space provided $\lambda(X)=1$. The lo-net spaces are particularly cases of the $\Omega$-net spaces studied by Jerry Vaughan [12], and in fact, they are the well-ordered net spaces $(\S 5)$.

III. Lo-net spaces. In this section we present various properties of lo-net spaces and some fundamental applications of lo-net spaces.

THEOREM 3.1.

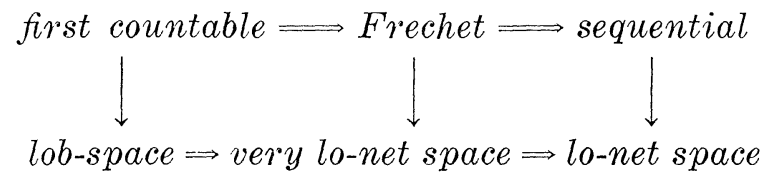

The quotient space obtained by attaching the 0 of the $1 / n$ sequence, $S_{1}$, to each countable ordinal in $\left[0, \omega_{1}\right]$ is a lo-net space which is not sequential and not very lo-net. Every lo-net closed set is sequentially closed, but sequentially closed does not imply lonet closed as $\left[0, \omega_{1}\right)$ is sequentially closed in $[0, \omega]$ but not closed. However in countable sets we have the following.

ThEOREM 3.2. Every countable sequentially closed set is lo-net closed.

Proof. Let $H$ be a countable sequentially closed set, and let $\left\{p_{\lambda}: \lambda \in L\right\}$ be a lo-net in $H$ where $p_{\lambda} \rightarrow p$. From Lemma 5.1, let $W$ be a well-ordered cofinal subset of $L$. Since $H$ is countable, if 
cf $(W)>\omega,\left\{p_{\lambda}: \lambda \in L\right\}$ must be constant on a cofinal subset of $W$. Since there is a constant subnet converging to $p, p \in H$. If cf $(W)=\omega$, choose an increasing sequence $\left\{\lambda_{n}: n \in \omega\right\}$ which is cofinal in $W$. Then since $\left\{p_{\lambda_{n}}: n \in \omega\right\}$ is cofinal in $\left\{p_{\lambda}: \lambda \in L\right\}, p_{\lambda_{n}} \rightarrow p$. Since $H$ is sequentially closed, $p \in H$. Thus $H$ contains each of its lo-net limit points and is lo-net closed.

CoRollary 3.3. Every countable lo-net space is sequential and every countable very lo-net space if Frechet.

COROLLARY 3.4. If $\left\{x_{n}\right\}$ is a sequence in a lo-net space and $\left\{x_{n}: n \in N\right\}$ is not closed, then $\left\{x_{n}\right\}$ has a convergent subsequence.

The mapping properties of lo-net spaces are as follows.

THEOREM 3.5. $X$ is a lo-net space (very lo-net space) [lob-space] if and only if $X$ is the quotient (pseudo-open) [open] image of alob-space.

THEOREM 3.6. The property of being a lo-net (very lo-net) [lob space] is preserved under quotient (pseudo-open) [open] mappings.

THEOREM 3.7. The class of lo-net spaces is a coreflective subcategory of the category of all topological spaces.

The next two theorems and Corollary 3.10 are generalizations of theorem 2.2 in [6] to arbitrary cardinals and lo-net spaces. An $m$-lo-net space is a lo-net space whose topology is generated by lonets with ranges of cardinality $\leqq m$. The character of the point $x$ in a space $X, \chi(x, X)$ is the least cardinal $\alpha$ for which there is a nhood base at $x$ of cardinality $\leqq \alpha$. The character of $X, \chi(X)=$ $\sup \{\chi(x, X): x \in X\}$. The pseudocharacter of the point $x$ in a space $X, \psi(x, X)$ is the least cardinal $\alpha$ such that $\{x\}$ is the intersection of $\leqq \alpha$ open sets.

THEOREM 3.8. Let $x$ be a non-isolated point in an lob-space $X$. $\chi(x, X) \leqq m$ if and only if there exist $M \subset X-\{x\}$ such that $\operatorname{card}(M) \leqq m$ and $x \in \operatorname{cl}(M)$.

Proof. We will prove only the sufficiency. Let $\{x\}$ be not open and let $M \subset X-\{x\}$ be such that $\operatorname{card}(M) \leqq m$ and $x \in \operatorname{cl}(M)$. Let $\left(\mathbb{U}_{x}\right.$ be a linearly ordered base at $x$. For each $p \in M$, let $U_{p} \in \mathbb{U}_{x}$ be such that $p \notin U_{p}$. If $\left\{U_{p}: p \in U\right\}$ is not cofinal in $\mathbb{U}_{x}$, then there is $U \in(\mathbb{U})_{x}$ such that $U \subset U_{p}$ for each $p \in M$ and thus $U \cap M=\varnothing$. Thus $\left\{U_{p}: p \in M\right\}$ is cofinal and $\chi(x, X) \leqq m$ which completes the proof. 
COROLLARY 3.9. In a lob-space, the following are equivalent,

(a) $\chi(X) \leqq m$

(b) $X$ is an m-lo-net space

(c) $t(X) \leqq m$

(d) If $\{x\}$ is not open, then there exists a set $M \subset X-\{x\}$ such that $\operatorname{card}(M) \leqq m$ and $x \in \operatorname{cl}(M)$.

TheOREM 3.10. If $X$ is a $T_{1}$ lob space, $\chi(x, X) \leqq m$ if and only if $\psi(x, X) \leqq m$.

Proof. The sufficiency is proven. Let $\left\{G_{\alpha}: \alpha \in A\right\}$ be a collection of open sets such that card $(A) \leqq m$ and $\{x\}=\cap\left\{G_{\alpha}: \alpha \in A\right\}$. Let $\mathbb{U}_{x}$ be a linearly ordered nhood base at $x$. For each $\alpha \in A$, choose $U_{\alpha} \in \mathbb{U}_{x}$ such that $U_{\alpha} \subset G_{\alpha}$. If $\left\{U_{\alpha}: \alpha \in A\right\}$ is not cofinal in $\mathbb{U}_{x}$, there is a $U_{\alpha} \in \mathbb{U}_{x}$ such that $U \subset U_{\alpha} \subset G_{\alpha}$, for each $\alpha \in A$. Thus $U=\{x\}$ and $\chi(x, X)=\omega \leqq m$. If $\left\{U_{\alpha}: \alpha \in A\right\}$ is cofinal, then it is a nhood base at $x$ of cardinality $\leqq m$ and $\chi(x, X) \leqq m$ and this completes the proof.

The next theorem improves both Theorem 2.3 of [6] and Proposition 1.1 of [10], and supplies a different view of a similar theorem in [8].

THEOREM 3.11. A $T_{1}$ lo-net space $X$ is sequentially compact if and only if it is countably compact.

Proof. We proof the sufficiency. Let $\left\{x_{n}\right\}$ be a sequence in $X$. If $\left\{x_{n}: n \in N\right\}$ is closed, then it is countable and compact. Hence $\left\{x_{n}: n \in N\right\}$ is first countable. If $\left\{x_{n}: n \in N\right\}$ is finite there is a convergent subsequence. If $\left\{x_{n}: n \in N\right\}$ is infinite there is a cluster point in $\left\{x_{n}: n \in N\right\}$ and a subsequence converges to it. If $\left\{x_{n}: n \in N\right\}$ is not closed, then it is not lo-net closed. Since countable sequentially closed sets are lo-net closed, by Theorem 3.2, $\left\{x_{n}: n \in N\right\}$ is not sequentially closed. Thus there is a sequence $\left\{x_{n_{k}}\right\}$ in $\left\{x_{n}\right\}$ that converges to some $p \notin\left\{x_{n}: n \in N\right\}$. Thus $X$ is sequentially compact and this completes the proof.

CoRollary 3.12. If $X_{\alpha}$ is a $T_{1}$ countably compact lo-net space for each $\alpha \in \omega_{1}$, the $\Pi\left\{X_{\alpha}: \alpha \in \omega_{1}\right\}$ is countably compact.

Corollary 3.13. If $X$ is a $T_{2}$ countably compact lo-net space and $\psi(X) \leqq c$, then $\operatorname{card}(X) \leqq c$.

IV. Further Applications. The introductory lemma in this section is the essential tool used in the applications of lo-nets to 
various weak covering theorems. The published proof of Theorem 2.4 [6] was modified to be an argument using a linearly ordered base. However, the unpublished proof of this theorem in the preprint of [6] is particularly relevant to the lo-net argument. A variation of this construction is presented here as the proof of the following lemma. It is given here for completeness and because it shows clearly the essential interaction between lo-nets and closures of unions which are at the center of the applications that follow.

LEMMA 4.1. If $\left(x_{\lambda}\right)_{\lambda \in L}$ is a lo-net in $\cup(G)$ with $x_{\lambda} \rightarrow x$, then either there is some $G \in(G)$ where $x \in \operatorname{cl}(G)$ or there is a subcollection $(G) \subset(G)$ and a choice function $y$ on (G) such that $x \in \operatorname{cl}\left(\left\{y(G): G \in @{ }^{\prime}\right\}\right)$.

Proof. Suppose $x \notin \operatorname{cl}(G)$ for each $G \in$ G. Well order (G). Let $\lambda_{1} \in L$ and let $G_{1}$ be the first set that contains $x_{\lambda_{1}}$. Suppose for each $\alpha<\beta, \lambda_{\alpha}$ and $G_{\alpha}$ are such that

(1) if $x_{\lambda} \in \mathbf{U}_{r<\alpha} G_{\gamma}$, then $\lambda<\lambda_{\alpha}$,

(2) $x_{\lambda_{\alpha<}} \notin \mathbf{U}_{r<\alpha} G_{\gamma}$ and

(3) $G_{\alpha}$ is the first set such that $x_{\lambda_{\alpha}} \in G_{\alpha}$.

Of particular importance to the definition of the choice function is the following: if $\eta<\nu$, then $x_{\lambda_{\nu}} \notin \mathbf{U}_{\gamma_{<\nu}} G_{\gamma}$. Thus, $x_{\lambda_{\nu}} \notin G_{\eta}$ and $x_{\lambda_{\nu}} \in G_{\nu}$. Hence for $\eta \neq \nu, G_{\eta}=G_{\nu}$. If $\left\{\lambda_{\alpha}: \alpha<\beta\right\}$ is cofinal in $L$, then $x_{\lambda_{\alpha}} \rightarrow x$ and we are finished by letting $(G)^{\prime}=\left\{G_{\alpha}: \alpha<\beta\right\}$ and let $y\left(G_{\alpha}\right)=x_{\lambda_{\alpha}}$, for each $\alpha<\beta$. Then $y$ is a choice function on (G) such that $x \in$ $\operatorname{cl}\left(\left\{y(G): G \in @{ }^{\prime}\right\}\right)$.

If $\left\{\lambda_{\alpha}: \alpha<\beta\right\}$ is not cofinal in $L$ we continue as follows. If $\beta=\nu+1$, then by hypothesis $G_{\nu}$ is the least set such that $x_{\lambda_{\nu}} \in G_{\nu}$, $x_{\lambda_{\nu}} \notin \bigcup_{\gamma_{<\nu}} G_{\gamma}$ and if $x_{\lambda} \in \bigcup_{\gamma_{<\nu}} G_{\gamma}$ then $\lambda<\lambda_{\nu}$. Since $x \notin \operatorname{cl}\left(G_{\nu}\right),\left\{\lambda: x_{2} \in G_{\nu}\right\}$ is bounded. Let $\lambda_{\beta} \in L$ be such that $\lambda<\lambda_{\beta}$, for each $x_{\lambda} \in G_{\nu}$, and let $G_{\beta}$ be the first set such that $x_{\lambda_{\beta}} \in G_{\beta}$. If $x_{\lambda} \in \mathbf{U}_{r_{<\beta}} G_{\gamma}=$ $\left(\bigcup_{i<\nu} G_{\gamma}\right) \cup G_{\nu}$, then $\lambda<\lambda_{\nu}<\lambda_{\beta}$ or $x_{\gamma} \in G_{\nu}$ and $\lambda<\lambda_{\beta}$ and in either case $\lambda<\lambda_{\beta}$. Thus $x_{\lambda_{\beta}} \notin \mathbf{U}_{i<\beta} G_{\gamma}$. Otherwise, if $\beta$ is a limit ordinal, let $\lambda_{\beta}$ be any index such that $\lambda_{\alpha}<\lambda_{\beta}$ for each $\alpha<\beta$ and let $G_{\beta}$ be the first set such that $\lambda_{\alpha}<\lambda_{\beta}$ for each $\alpha<\beta$ and let $G_{\beta}$ be the first set such that $x_{\lambda_{\beta}} \in G_{\beta}$. If $x_{\lambda} \in \mathbf{U}_{\alpha<\beta} G_{\alpha}$, then $x_{\lambda} \in G_{\alpha_{0}}$ for $\alpha_{0}<\beta$. Thus, $x_{\lambda} \in \bigcup\left\{G_{\alpha}: \alpha<\alpha_{0}+1\right\}$ and $\lambda<\lambda_{\alpha_{0}+1}<\lambda_{\beta}$. Also, if $x_{\lambda_{\beta}} \in G_{\gamma}$ for $\gamma<\beta$, then $\lambda_{\beta}<\lambda_{\gamma}<\lambda_{\beta}$ and this contradiction implies $x_{\lambda_{\beta}} \notin \mathbf{U}_{r<\beta} G_{\gamma}$. Thus appropriate $\lambda_{\beta}$ and $G_{\beta}$ are selected in either case. Since $\beta$ is bounded by card $(L)^{+}$, the induction continues until a cofinal case is reached. This completes the proof.

Theorem 2.4 [6] is the key theorem for the many applications of lob spaces to the various weak covering theorems in [6] and [7]. This important theorem is true for the weaker notion of very lo-net spaces and is restated here as a consequence of Lemma 4.1. 
THEOREM 4.2. [Davis] If $X$ is a very lo-net space and $U$ is a collection of subsets such that $p \in \mathrm{cl}(\cup @)$ then either there exists a $G \in(G)$ such that $x \in \mathrm{cl}(G)$ or there exists (G)' $\subset$ (G) and a choice function $y$ on $(G)$ such that $\left.x \in \operatorname{cl}\left(\{y(G): G \in \mathbb{G})^{\prime}\right\}\right)$.

Thus this class of pseudo-open images of lob-spaces suffices to insure the validity of many results found in [6] and [7]. The natural question is then if the weaker lo-net spaces suffice to prove Theorem 4.1. The counterexample is extremely simple and is a well known lo-net space.

EXAMPLE 4.3. Theorem 4.1 is not true for lo-net spaces.

Consider the space $S_{2}=\{(0,0)\} \cup\{(1 / n, 0): n \in N\} \cup\{(1 / n, 1 / m)$ : $n, m \in N\}$ in [1] and [4]. Let $U_{n}=\{(1 / n, 1 / m): m \in N\}$. Then $(0,0) \notin$ $\operatorname{cl}\left(U_{n}\right)$ for each $n$ and any choice function $y$ on (U) $=\left\{U_{n}: n \in N\right\}$ selects one point $y\left(U_{n}\right)=p_{n}$ in each $U_{n}$. But $(0,0) \notin \operatorname{cl}\left(\left\{p_{n}: n \in N\right\}\right)$.

A space $X$ is called a quasi $k$-space if a subset $H \subset X$ is closed if and only if $F \cap C$ is closed in $C$ for every countably compact subspace $C \subset X$. Theorem 4.2 has an interesting companion theorem; Lemma 2.3.1 of [7]. However, Lemma 2.3.1 is false as the previous example shows. $S_{2}$ is sequential and thus is a $k$-space. Hence $S_{2}$ is a quasi-k-space. Since $(0,0) \in \operatorname{cl}\left(\cup(\mathbb{U}) \backslash \cup\left\{\operatorname{cl}\left(U_{n}\right): n \in N\right\},\left\{U_{n}: n \in N\right\}\right.$ is not closure preserving. Any set formed by choosing a finite number of points in each $U_{n}$ has no limit points. In particular, the choice function must be defined on the closures of the sets in some subcollection $\left(\mathbb{C}^{\prime}\right.$. This is a subtle point relating to the weak topology induced by a class of sets and is extremely important here. A corrected version of this lemma follows.

THEOREM 4.4. If $X$ is a quasi k-space and (G) is a collection of subsets which is not closure preserving, then there is a subcollection $(G) \subset$ (G) and a choice function $y$ on $\{\mathrm{cl}(G): G \in(G\}$ such that $\{y(\mathrm{cl}(G))$ : $\left.G \in \mathbb{G}^{\prime}\right\}$ has a cluster point.

Proof. For some subcollection (G) $\subset @, \cup\left\{\operatorname{cl}(G): G \in \Theta^{*}\right\}$ is not closed. Then there exists a countably compact set $C$ such that $C \cap$ ( $\cup\left\{\mathrm{cl}(G): G \in \mathbb{G}^{*}\right\}$ ) is not closed in $C$. Let $G^{*}$ be well ordered, using an initial set of ordinals, and let $\nu$ be the least ordinal such that $C \cap\left(\cup\left\{\mathrm{cl}\left(G_{\alpha}\right): \alpha<\nu\right\}\right)$ is not closed in $C$. Then $\nu$ is a limit ordinal and for each $\alpha$ in a cofinal subset of $[1, \nu)$, there is a point $x_{\alpha} \in\left(C \cap \mathrm{cl}\left(G_{\alpha}\right)\right) \backslash \cup\left\{\mathrm{cl}\left(G_{\beta}\right): \beta<\alpha\right\}$. Choose this $x_{\alpha}=y\left(\operatorname{cl}\left(G_{\alpha}\right)\right)$ from each $\mathrm{cl}\left(G_{\alpha}\right)$ for this cofinal set of $[1, \nu)$ and let $(G)$ be the subcollec- 
tion of (a) is lexed by this cofinal set. Thus, $\left\{y(\operatorname{cl}(G)): G \in G^{\prime}\right\}$ is an infinite subset of $C$ and thus has a cluster point. This completes the proof.

Of additional interest is the following modified extension of theorem 4.2. This extension to the lo-net spaces is a parallel theorem to the previous theorem 4.4. This is parallel in the sense that this theorem also uses the weak topology induced by a collection of sets, namely the lo-nets, as theorem 4.4 used the countably compact subspaces. However, it is completely independent because Examples 3.7 and 3.8 of Davis [6] also show there is no subclass relationship between lo-net spaces and quasi $k$-spaces.

THEOREM 4.5. If $X$ is a lo-net space and (a) is a collection of subsets of $X$ which is not closure preserving, then there is a subcollection (G)' $\subset$ (G) and a choice function $y$ on $\left\{\mathrm{cl}(G): G \in \Theta^{\prime}\right\}$ such that $\left.\{y(\mathrm{cl}(G)): G \in G)^{\prime}\right\}$ has a cluster point.

Proof. For a subcollection (G)* $\subset\left(G, \cup\left\{\mathrm{cl}(G): G \in \Theta^{*}\right\}\right.$ is not closed. Since $X$ is a lo-net space, there exists a lo-net $\left(x_{2}\right)$ in $\cup\left\{\mathrm{cl}(G): G \in \mathbb{G}^{*}\right\}$ converging to a point $x$ which is not in $\cup\{\mathrm{cl}(G)$ : $\left.G \in \mathbb{G}^{*}\right\}$. Thus, $x \notin \mathrm{cl}(G)$, for each $G \in G^{*}$ and by the selection process in Lemma 4.1 and $\left[6\right.$, Th. 2.4] there exists $G^{\prime} \subset G *$ and a choice function $y$ on $\left\{\mathrm{cl}(G): G \in @{ }^{\prime}\right\}$ such that $\left.x \in \operatorname{cl}(\{y(\operatorname{cl}(G)): G \in @)\}\right)$. Thus $\left\{y(\mathrm{cl}(G)): G \in \mathbb{G}^{\prime}\right\}$ has a cluster point and this completes the proof.

The following definitions are due to Briggs [5]. A collection of subsets of a space $X, A)=\left\{H_{\alpha}: \alpha \in A\right\}$ is a ppc-collection (W-ppccollection) provided: if $B \subset A$ is infinite (uncountable) and if $p_{\beta}$ and $q_{\beta} \in H_{\beta}$ for each $\beta \in B$ and $\alpha \neq \beta$ implies $p_{\alpha} \neq p_{\beta}$ and $q_{\alpha} \neq q_{\beta}$, then $Q=\left\{q_{\beta}: \beta \in B\right\}$ has a cluster point whenever $P=\left\{p_{\beta}: \beta \in B\right\}$ has a cluster point. A space is preparacompact ( $\mathbf{K}$-preparacompact) if every open cover has a ppc-refinement ( $\boldsymbol{\aleph}$-pp-refinement).

Since theorems 4.4 and 4.5 are true for the collection of closures, we add further that $(A)$ will be called a strong ppc-collection (strong $\gamma$-ppc-collection) if the $p_{\beta}$ and $q_{\beta}$ can be chosen from the closures of the sets $H_{\beta}$. Also, a space will be strongly preparacompact (strongly $\boldsymbol{\aleph}$-preparacompact) if every open cover has a strong ppcrefinement (strong $\boldsymbol{\aleph}$-ppc-refinement). These notions were independently introduced and studied by Nitta [11]. Observe in Example 4.2 the collection $\left\{U_{n}: n \in N\right\}$ is a ppc-collection which is not a strong ppc-collection.

THeOREM 4.6. [Davis [6]] Let $X$ be a very lo-net space and let (ii) $=\left\{G_{\alpha}: \alpha \in A\right\}$ be an $\aleph$-ppc collection of open subsets of $X$. If there 
exists a discrete collection $\left\{D_{\beta}: \beta \in B\right\}$ of nonempty subsets of $X$ such that $D_{\beta} \subset G_{\beta}$ for $\beta \in B \subset A$, then $\left\{G_{\beta}: \beta \in B\right\}$ is either countable or closure preserving.

Theorem 4.6 is not true for lo-net spaces as the following example shows.

EXAMPLE 4.7. Let $T$ be the set of countable nonlimit ordinals. For each $\beta \in T$, let $S_{1}(\beta)$ be a copy of the convergent $1 / n$-sequence with limit point $0_{\beta}$. Let $X$ be the quotient space formed by attaching the limit point $0_{\beta}$ of $S_{1}(\beta)$ to $\beta$ in the ordinal space $\left[0, \omega_{1}\right]$, for each $\beta \in T . \quad X$ is a lo-net space. If $G_{\beta}=S_{1}(\beta)-\left\{0_{\beta}\right\}$, for each $\beta \in T$, (G) $=\left\{G_{\beta}: \beta \in T\right\}$ is an $\aleph$-ppc-collection. If $D_{\beta}$ consists of any finite subset of $G_{\beta}$ for each $\beta \in T$, then $\left\{D_{\beta}: \beta \in T\right\}$ is discrete. However, $\left\{G_{\beta}: \beta \in T\right\}$ is neither countable nor closure preserving.

Theorem 4.6 has the following valid variation for lo-net spaces.

THEOREM 4.8. Let $X$ be a lo-net space (or a quasi k-space) and let $G=\left\{G_{\alpha}: \alpha \in A\right\}$ be a strongly $\aleph$-ppc-collection of subsets of $X$. If there exists a discrete collection $\left\{D_{\beta}: \beta \in B\right\}$ of nonempty subsets of $X$ such that $D_{\beta} \subset G_{\beta}$, for each $\beta \in B \subset A$, then $\left\{G_{\beta}: \beta \in B\right\}$ is either countable or closure preserving.

Proof. Let $B$ be uncontable and suppose $\left\{G_{\beta}: \beta \in B\right\}$ is not closure preserving. By theorem 4.5 (or theorem 4.4) there is a subset $B_{1} \subset B$ and a choice function $y$ on $\left\{\operatorname{cl}\left(G_{\beta}\right): \beta \in B_{1}\right\}$ such that $\left\{y\left(\operatorname{cl}\left(G_{\beta}\right)\right): \beta \in B_{1}\right\}$ has a cluster point. Let $B_{2} \subset B_{1}$ be such that for distinct elements $\alpha, \beta \in B_{2}, y\left(\operatorname{cl}\left(G_{\alpha}\right)\right) \neq y\left(\operatorname{cl}\left(G_{\beta}\right)\right)$. For each $\beta \in B$, choose any $q_{\beta} \in D_{\beta}$. For $\beta \in B_{2}$, let $p_{\beta}=y\left(\operatorname{cl}\left(G_{\beta}\right)\right)$ and for $\beta \in B-B_{2}$, let $p_{\beta}=q_{\beta}$. Then $\left\{q_{\beta}: \beta \in B\right\}$ is a closed discrete set, but $\left\{p_{\beta}: \beta \in B\right\}$ has a cluster point. This contradicts the fact that (G) is a strong $\$$-ppc-collection. This proves the theorem.

Accordingly, the following variation of [6, Th. 3.3] is a valid characterization of paracompactness in either lo-net spaces or quasi$k$-spaces.

THEOREM 4.9. If $X$ is a regular lo-net space (or quasi k-space), then $X$ is paracompact if and only if $X$ is irreducible and strongly X-preparacompact.

Also, if $H$ is an $\aleph$-ppc collection and $\mathrm{cl}\left(G_{\alpha}\right) \subset H_{\alpha}$ for each $\alpha \in A$, then $\left\{\mathrm{cl}\left(G_{\alpha}\right): \alpha \in A\right\}$ is an $\boldsymbol{K}$-ppc-collection. Thus Lemma 3.4.2 [7] which uses quasi-k-spaces can also be stated for lo-net spaces and 
its validity follows from Theorem 4.8.

TheOREM 4.10. Let $X$ be a lo-net space and let $\mathbb{H}=\left\{H_{\alpha}: \alpha \in A\right\}$ be an $\aleph$-ppc-collection such that for the collection $\left\{G_{\alpha}: \alpha \in A\right\}, \operatorname{cl}\left(G_{\alpha}\right) \subset H_{\alpha}$ for each $\alpha \in A$. If there exists a discrete collection $\left\{D_{\beta}: \beta \in B \subset A\right\}$ of nonempty subsets such that $D_{\beta} \subset G_{\beta}$, for each $\beta \in B$, then $\left\{G_{\beta}\right.$ : $\beta \in B\}$ is either countable or closure preserving.

With ppc in place of $\boldsymbol{K}$-ppc the word countable may be omitted from the conclusion.

Sheldon Davis has been gracious enough to carefully study the preprint of this paper and has supplied the following results, which extend various theorems in [7]. The proofs are easily excessible as modifications of those in [7] and are omitted here.

THEOREM 4.11. If $X$ is a normal (regular) preparacompact (or $\aleph$-preparacompact) lo-net space, then $X$ is collectionwise normal (Hausdorff).

CoRollary 4.12. If (P) is property such that (P) plus collectionwise normality implies paracompact, then every normal, preparacompact lo-net space which satisfies (P) is paracompact.

CoRollary 4.13. If $X$ is a normal lo-net space, then $X$ is paracompact if and only if $X$ is preparacompact and $\theta$-refinable.

CoRollary 4.14. If $X$ is a normal lo-net space and $X=\cup\left\{F_{n}\right.$ : $n \in \omega\}$ where $F_{n}$ is closed and isocompact for each $n \in \omega$, then $X$ is isocompact (See Th. 2.6 of [7].)

COROLLARY 4.15. Isoparacompactness is $F_{\sigma}$-hereditary in normal lo-net spaces.

THEOREM 4.16. If $Y$ is a preparacompact lo-net space and $f: X \rightarrow Y$ is closed, continuous, finite-to-one, then $X$ is paracompact. (Proof of Th. 2.5 [7] suffices.)

Corollary 4.17. If $Y$ is a lo-net space, $f: X \rightarrow Y$ is closed continuous, finite to one and $X$ is isocompact, then $Y$ is isocompact.

CoRollary 4.18. If $X=\cup\left\{X_{\alpha}: \alpha \in A\right\}$ is a lo-net space where $\left\{X_{\alpha}: \alpha \in A\right\}$ is a closed, locally finite collection and $X_{\alpha}$ is isoparacompact for each $\alpha \in A$, then $X$ is isoparacompact. 
THEOREM 4.19. Every preparacompact lo-net space is ppc-expandable. (See Th. 3.2. [7].)

THEOREM 4.20. If $X$ is a normal ppc-expandable lo-net space, then any locally finite collection of closed sets has a ppc-expansion.

THEOREM 4.21. If $X$ is a normal (regular) discretely ppcexpandable lo-net space, then $X$ is collectionwise normal (Hausdorff).

V. Well-ordered net spaces. In this section the true nature of lo-net spaces as a generalization of sequential spaces and lobspaces, is revealed in the characterization, Theorem 5.2. The following fact is essential.

Lemma 5.1. For each linearly ordered set, there is a well-ordered cofinal subset.

This lemma implies that the lob-spaces of Davis [6] are precisely the spaces which have well-ordered local bases, wob-spaces (well ordered by reverse inclusion). The quotient spaces of wob-spaces would be characterized as those spaces which have the weak topology generated by the collection of well-ordered nets. Thus, I define a space $X$ to be a well-ordered net space (wo-net space) provided $X$ has the weak topology generated by the natural cover of wellordered nets in $X$. Since lob-space $\equiv$ wob space, the classes of quotient spaces are identical.

THEOREM 5.2. A space is a lo-net space if and only if it is a wo-net space.

The properties of lo-net spaces presented in $\S 3$ should be reexamined as wo-net space properties. The class of test spaces for the wo-net spaces (thus lo-net spaces has been determined in [3] as the test spaces for sequential spaces were determined in [2].

I would like to express my gratitude to the referee for suggestions which have improved this paper.

\section{REFERENCES}

1. A. Arkangelskii and S. P. Franklin, Ordinal invariants for topological spaces, Michigan Math. J., 15 (1968), 313-320.

2. S. A. Baber and J. R. Boone, Test spaces for infinite sequential order, submitted. 3. - Test spaces for $\Omega$-net spaces, in processing.

4. J. R. Boone, S. W. Davis and G. Gruenhage, Cardinal functions for k-spaces, Proceedings of AMS, 68 (1978), 355-358. 
5. R. C. Briggs, Preparacompactness and $\boldsymbol{\aleph}$-preparacompactness in q-spaces, Colloquium Mathematicum, 27 (1973), 227-235.

6. S. W. Davis, Spaces with linearly ordered bases, Topology Proceedings 3 (1978), 37-51.

7. S. W. Davis and S. C. Smith, The paracompactness of preparacompact spaces, to appear in Topology Proceedings.

8. K. M. Dvei, P. R. Meyer and M. Rajagopalan, When does contable compactness imply sequential compactness?, General Topology and its Applications, 6 (1976), 279-289.

9. S. P. Franklin, Natural covers, Compositio Mathematica, 21 (1969), 253-261.

10. - Spaces in which sequences suffice, Fundamenta mathematica, 57 (1975), 107-115.

11. S. Nitta, Strong-preparacompactness in quasi-k-spaces, Mathematica Japanicae, 19 (1974), 291-296.

12. J. E. Vaughan, Convergence, closed projections and compactness, Proceedings of AMS, 51 (1975), 496-476.

Received September 3, 1980 and in revised form January 6, 1981.

Texas A \& M University

College Station, TX 77843 



\title{
PACIFIC JOURNAL OF MATHEMATICS
}

\section{EDITORS}

\author{
DONALD BABBITT (Managing Editor) \\ University of California \\ Los Angeles, CA 90024 \\ Hugo RossI \\ University of Utah \\ Salt Lake City, UT 84112 \\ C. C. Moore and ANDrew OGG \\ University of California \\ Berkeley, CA 94720
}

\section{J. DugundjI}

Department of Mathematics

University of Southern California

Los Angeles, CA 90007

R. FinN and J. Milgram

Stanford University

Stanford, CA 94305

\section{ASSOCIATE EDITORS}
R. ARENS
E. F. BeCKENBACH
B. H. NeUMANN
F. WOLF
K. YoshidA

\section{SUPPORTING INSTITUTIONS}

UNIVERSITY OF ARIZONA

UNIVERSITY OF BRITISH COLUMBIA

CALIFORNIA INSTITUTE OF TECHNOLOGY

UNIVERSITY OF CALIFORNIA

MONTANA STATE UNIVERSITY

UNIVERSITY OF NEVADA, RENO

NEW MEXICO STATE UNIVERSITY

OREGON STATE UNIVERSITY

\author{
UNIVERSITY OF OREGON \\ UNIVERSITY OF SOUTHERN CALIFORNIA \\ STANFORD UNIVERSITY \\ UNIVERSITY OF HAWAII \\ UNIVERSITY OF TOKYO \\ UNIVERSITY OF UTAH \\ WASHINGTON STATE UNIVERSITY \\ UNIVERSITY OF WASHINGTON
}

The Supporting Institutions listed above contribute to the cost of publication of this Journal, but they are not owners or publishers and have no responsibility for its content or policies.

Mathematical papers intended for publication in the Pacific Journal of Mathematics should be in typed form or offset-reproduced, (not dittoed), double spaced with large margins. Please do not use built up fractions in the text of the manuscript. However, you may use them in the displayed equations. Underline Greek letters in red, German in green, and script in blue. The first paragraph or two must be capable of being used separately as a synopsis of the entire paper. Please propose a heading for the odd numbered pages of less than 35 characters. Manuscripts, in triplicate, may be sent to any one of the editors. Please classify according to the scheme of Math. Reviews, Index to Vol. 39. Supply name and address of author to whom proofs should be sent. All other communications should be addressed to the managing editor, or Elaine Barth, University of California, Los Angeles, California, 90024.

50 reprints to each author are provided free for each article, only if page charges have been substantially paid. Additional copies may be obtained at cost in multiples of 50 .

The Pacific Journal of Mathematics is issued monthly as of January 1966. Regular subscription rate: $\$ 102.00$ a year (6 Vols., 12 issues). Special rate: $\$ 51.00$ a year to individual members of supporting institutions.

Subscriptions, orders for numbers issued in the last three calendar years, and changes of address shoud be sent to Pacific Journal of Mathematics, P.O. Box 969, Carmel Valley, CA 93924, U.S.A. Old back numbers obtainable from Kraus Periodicals Co., Route 100, Millwood, NY 10546.

PUBLISHED BY PACIFIC JOURNAL OF MATHEMATICS, A NON-PROFIT CORPORATION

Printed at Kokusai Bunken Insatsusha (International Academic Printing Co., Ltd.). 8-8, 3-chome, Takadanobaba, Shinjuku-ku, Tokyo 160, Japan. 


\section{Pacific Journal of Mathematics}

\section{Vol. 98, No. 1 \\ March, 1982}

Humberto Raul Alagia, Cartan subalgebras of Banach-Lie algebras of

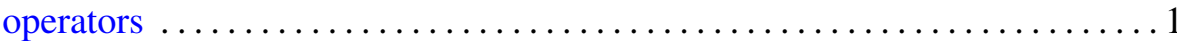

Tom M. (Mike) Apostol and Thiennu H. Vu, Elementary proofs of

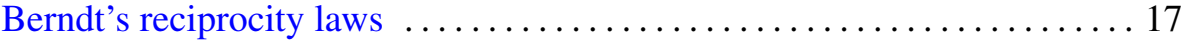

James Robert Boone, A note on linearly ordered net spaces $\ldots \ldots \ldots \ldots . \ldots 25$

Miriam Cohen, A Morita context related to finite automorphism groups of

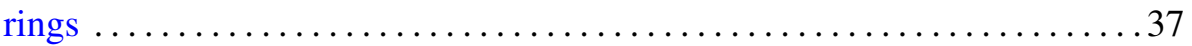

Willibald Doeringer, Exceptional values of differential polynomials . . . . . . 55

Alan Stewart Dow and Ortwin Joachim Martin Forster, Absolute

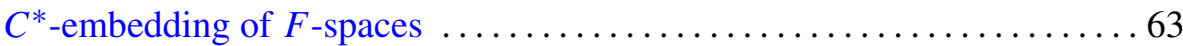

Patrick Hudson Flinn, A characterization of $M$-ideals in $B\left(l_{p}\right)$ for

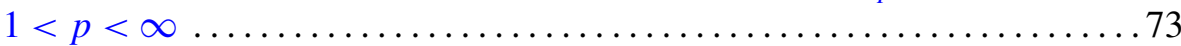

Jack Emile Girolo, Approximating compact sets in normed linear spaces . . . 81

Antonio Granata, A geometric characterization of $n$th order convex

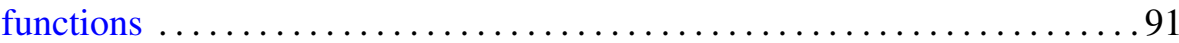

Kenneth Richard Johnson, A reciprocity law for Ramanujan sums . . . . . . .99

Grigori Abramovich Kolesnik, On the order of $\zeta\left(\frac{1}{2}+i t\right)$ and $\Delta(R) \ldots \ldots 107$

Daniel Joseph Madden and William Yslas Vélez, Polynomials that

represent quadratic residues at primitive roots $\ldots \ldots \ldots \ldots \ldots \ldots \ldots 123$

Ernest A. Michael, On maps related to $\sigma$-locally finite and $\sigma$-discrete

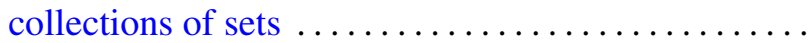

Jean-Pierre Rosay, Un exemple d'ouvert borné de $\mathbf{C}^{3}$ “taut" mais non

hyperbolique complet

Roger Sherwood Schlafly, Universal connections: the local problem

Russel A. Smucker, Quasidiagonal weighted shifts .....

Eduardo Daniel Sontag, Remarks on piecewise-linear algebra . .

Jan Søreng, Symmetric shift registers. II

H. M. (Hari Mohan) Srivastava, Some biorthogonal polynomials suggested

by the Laguerre polynomials 